\title{
Methods for rapid quantification of brain autoradiographs
}

\author{
J. MICHAEL WALKER, DAVID G. LAVOND, DAVID J. BODART \\ MICHAEL S. BEATTIE, and PATRICIA M. MEYER \\ Laboratory of Comparative and Physiological Psychology \\ Ohio State University, Columbus, Ohio 43212
}

\begin{abstract}
Apparatus and methods are described for rapid semiautomatic quantification of silver grain densities in brain autoradiographs. The output voltage of a photodiode used with darkfield oil microscopy reflects the amount of label in a 35 by 35 square micra area of brain tissue. Apparatus are described for monitoring the movement of a microscope stage, and a microprocessor interface is described that provides hard-copy and paper-tape output of grain densities for later processing.
\end{abstract}

Hughes, Meyer, Meyer, Meyer, and Bresnahan (1977) describe a simple and relatively inexpensive microphotometer system for measuring silver grain densities in autoradiographs. The basic components include a microscope with a $100 \mathrm{X}$ oil objective, an oil dark-field condenser, a photodiode with an operational amplifier, and a digital volt meter (DVM). Light reflected from silver grains in dark-field autoradiographic brain material passes through a camera tube of the microscope to the photodiode; the amplified photodiode signal indicates the grain density in millivolts, which is displayed on the DVM. The authors compare average millivolt readings with average grain counts in several areas of one brain section and report a highly significant correlation between millivolt output and grain counts $(r=+.95)$. The regression equation is $y=.01 x+2.69$, in which $\mathrm{y}$ is the millivolt reading and $\mathrm{x}$ is the actual grain count.

In the present article, we describe other equipment and procedures that facilitate the use of the microphotometer system described by Hughes et al. (1977). Using the Hughes et al. system in combination with the system described here, data that previously required $40 \mathrm{~h}$ to collect can be gathered in $2 \mathrm{~h}$. Furthermore, the system is inexpensive compared to commercial systems. The additional equipment includes an $X Y$ plotter, two longitudinal potentiometers attached to the $\mathrm{X}$ and $\mathrm{Y}$ axes of the microscope stage, and two precision voltage reference sources. The voltage reference source is an electronic interface between the mechanical stage of the microscope and the $X Y$ plotter so that movement of the plotter pen is coordinated with movement of the

This study was supported in part by National Institute of Mental Health Grant MH-06211. The first and third authors were predoctoral research assistants at the Ohio State University; the second author was a predoctoral University Fellow at the Ohio State University; the fourth author was a United States Health Service predoctoral fellow supported by Training Grant MH-06748; and the last author was a United States Public Health Service Research Development Investigator (5-K02-MH-12,747).
$X$ and $Y$ axes of the microscope stage. When examining extremely small units of tissue, this adjunct apparatus facilitates accuracy in moving from one specified region to another under high-power magnification. A microcomputer interface to a Teletype provides an easy means for recording the data in printed form and on paper tape. Methods are discussed in this paper for calibrating the microscope with the photometer system to assure the collection of reliable data that are comparable between different specimens.

\section{METHOD}

\section{Equipment}

Figure $1 \mathrm{~A}$ illustrates the arrangement of all the components in the microphotometer system. We use a Leitz microscope (Figure 1A and Figure 1B) equipped with lamp housing, Model 100 (Figure 1Bh) and a dc power supply (Figure 1A, Sola, Catalog Number 83-120310-1) for a 12-V 100-W tungsten halogen lamp. Not shown in the figure are a blue filter and a white heat filter within the lamp housing that prevent bleaching of stained tissue after prolonged exposure. Attached to the camera tube of the microscope is the photodiode (Figure 1Af and Figure $1 \mathrm{Bf}$ ), which is connected to a dc amplifier (United Detector Technology, Inc., Model UDT 101 A) mounted within the control box (Figure 1Ac and Figure 1C). An output from the amplifier is connected to a Weston DVM (Model 1234 276513), which is mounted in the top portion of the control box (Figure $1 \mathrm{Ag}$ and Figure 1C). The DVM is used both for displaying the output of the photodiode and for calibration of the microscope stage with the $\mathrm{XY}$ plotter. A precision potentiometer (Vernitron, 300 Marcus Boulevard, Deer Park, New York 11729, Catalog Number 15392, Type 113) is mounted on each of the $X$ and $Y$ areas of the microscope stage (Figures $1 \mathrm{Ae}$ and $1 \mathrm{Be}$ ); each is connected to a precision voltage reference source contained within the control box. Each voltage reference is then connected to a differential amplifier (Type 200) contained within the main frame of the Houston Model 2200-3-3 XY plotter (Figures 1Ad and 1D).

\section{Positioning Sy stem}

The electromechanical system accurately monitors movements of the microscope stage on the XY plotter to increments as small as $\mathbf{5 0}$ micra on the stage. The position of the stage is transduced into voltage by linear 3 -kohm potentiometers 
A

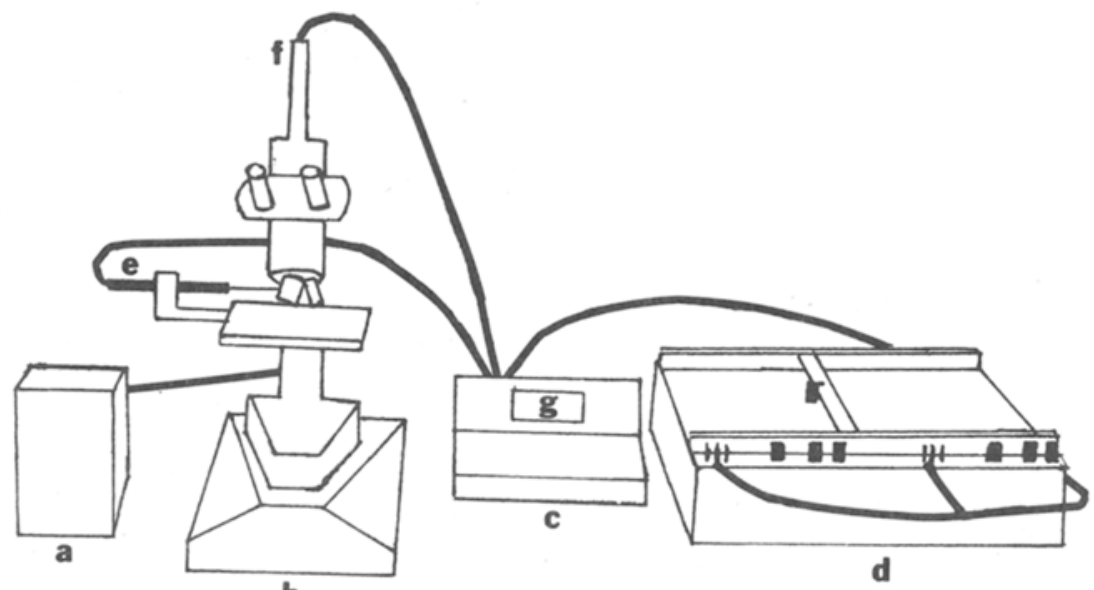

b
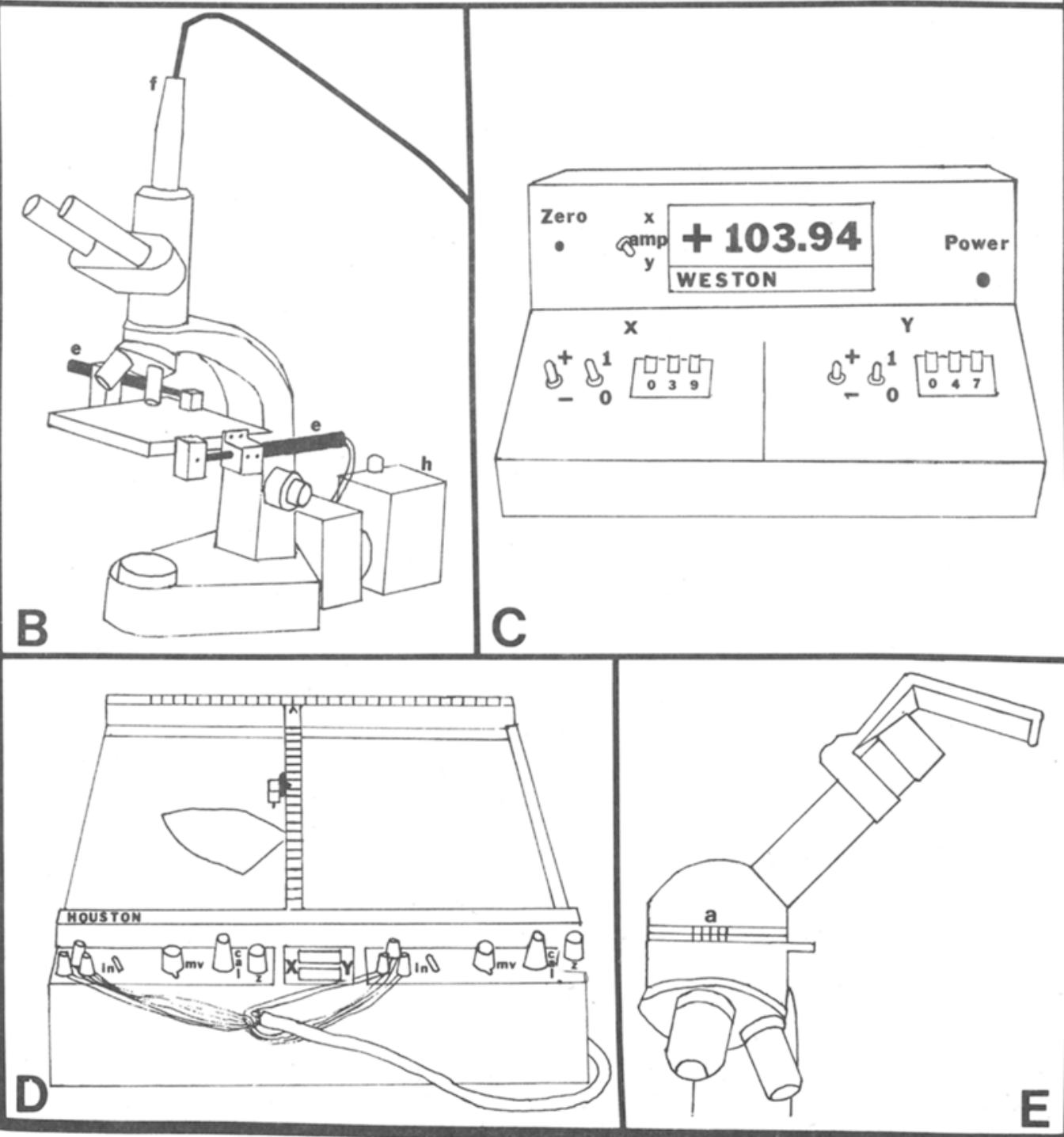

Figure 1. Components of the microprocessor system. 
mounted as shown in Figures 1 Ae and 1 Be. Potentiometer shaft length is $30.00 \mathrm{~mm}$, with $.5 \%$ linearity and $.05 \%$ smoothness. Each potentiometer is arranged as a voltage divider so that the output voltage is proportional to the position of the shaft and the applied voltage. Since $30 \mathrm{~V}$ is applied across each potentiometer, the output voltage changes by $.3 \mathrm{mV} / \mathrm{micron}$ of stage movement. This rate represents the ideal case, and in monitoring such small movements it is extremely important to be aware of the degree of error. For this reason, we checked the accuracy of the XY plotter for the small increments (50 micra) used in making measurements of tissue grain densities. A change in potentiometer output of $15.7 \mathrm{mV}$ corresponds to $\bar{x}=52.5 \pm 6.5 \mathrm{micra}$ and $\bar{y}=51.9 \pm 6.5 \mathrm{micra}$ (range $=30-65$ microns). For greater accuracy, the manufacturer (Vernitron) recommends Catalog Number $15392 \mathrm{~A}$, which limits the variability between readings even further.

Although we use 3-kohm potentiometers, the absolute resistive values are not critical, since the voltage division is independent of the total resistance. However, it is generally preferable to use a relatively small potentiometer resistance, since the output is in parallel with the plotter input resistance and with resistors that divide the potentiometer output voltage for input to the DVM. Therefore, the output voltage is in error by a factor determined by the paraliel sum of these resistances, making it important to have a very high input resistance in the measurement devices (the DVM and XY plotter) and a small resistance on the stage potentiometers.

Variations in the voltage applied to the potentiometer are transferred to the output of the potentiometers and appear as changes in position-voltage proportion, or as drift that may cause spontaneous movements of the plotter. We therefore use a commercially available reference supply designed for critical applications (Calex, Model 2.15.100, Pleasant Hill, California).

In operation, the sensitivity of the amplifiers in the $X Y$ plotter are set to $100 \mathrm{mV} /$ in. (Figure 1D) to observe the small voltage changes produced by the movements of the stage. Since the voltage across the stage potentiometers ranges from 0 to $30 \mathrm{~V}$, exceeding the potential space on the plotter, it is often necessary to oppose the resting voltage of the potentiometers with an adjustable voltage source to avoid saturation of the plotter amplifiers. The resting output of the potentiometers is divided by 1,000 , as illustrated in the circuit diagram in Figure 2, and may be displayed on the DVM. The value on the DVM multplied by 1,000 represents the bucking voltage needed to be applied to one input of the differential amplifiers on the plotter. By using the circuit in Figure 2, any voltage between $-15 \mathrm{~V}$ and $+15 \mathrm{~V}$ can be applied to the plotter.

Two operational amplifiers (National Semiconductor Model LH0044C), configured as summing inverters, and a reference supply form the adjustable source used to interface the potentiometer circuit to the plotter amplifiers. The operational amplifiers are instrumentation grade devices with low noise and low drift. The function of these units is to algebraically sum inputs that determine the sign and magnitude of the reference output. The .01 -microF capacitors between Pins 7 and 8 of each amplifier provide frequency compensation for unity gain; the amplifier requires no offset adjustment. The 100 -kohm resistors that close the feedback loop and enter the inverting input set the gain of the LH0044C to 1.00 and the input impedence at $100 \mathrm{kohm}$. The $49.9-\mathrm{kohm}$ resistor between the positive inputs and ground balance the amplifiers for the input bias current.

The bucking voltage input to the plotter is set with digital potentiometers (Bourne Model 36805-1-2k) linked to the reference supply through a resistive voltage divider. Calibration of the reference source is relatively simple. First, the 200-ohm trimming potentiometers (in series with "sign" switch) are

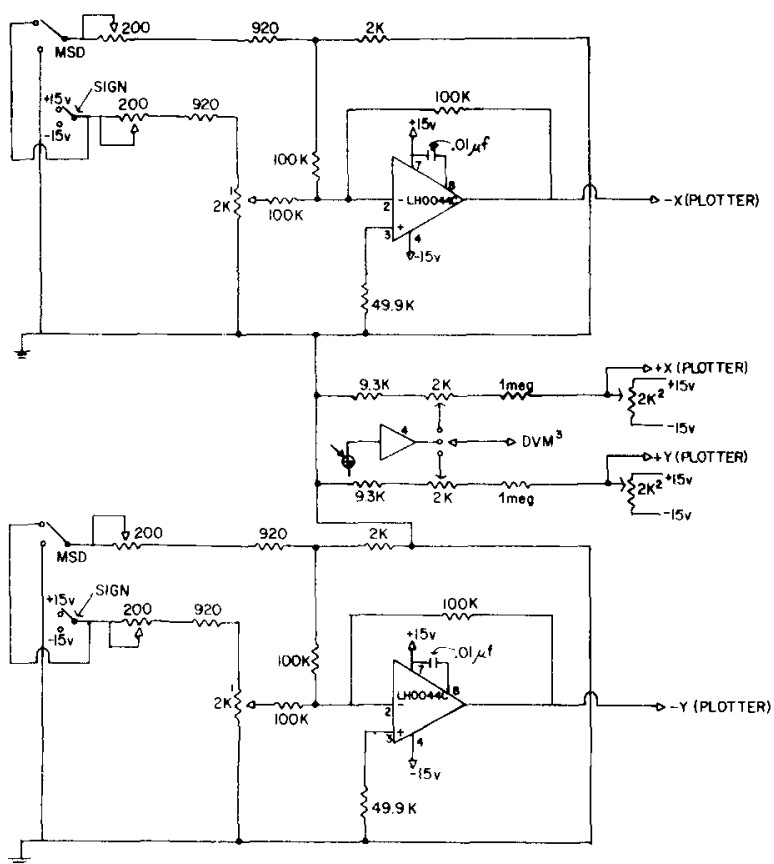

Figure 2. Schematic diagram of the voltage reference source used in monitoring movement of the microscope stage. Voltages are derived from Calex reference supply, Number 2.15.100. Labeled 1 in the diagram are the Bourns, Model 36805-1-2K, digital potentiometers. Labeled 2 are the linear stage-mounted potentiometers (Vernitech, Deer Park, New York, Model 15392; illustrated in Figures $1 \mathrm{Ae}$ and $1 \mathrm{Be})$. Labeled 3 is the DVM, Weston Digital Panel Meter, Model 1234-276513. Labeled 4 is the photodiode system previously described by Hughes et al. (1977).

adjusted so that each increment of the highest order digit of the digital potentiometer produces a pen movement of 1 in. on the plotter; plotter sensitivity is set at $1 \mathrm{~V} /$ in. Subsequently, this trimming potentiometer is adjusted with finer sensitivities of the plotter until the least significant digit on the digital potentiometer gives a change of exactly $10 \mathrm{mV}$. Next, the potentiometers $(200 \mathrm{ohm})$ in series with the most significant digit (MSD) switch are adjusted so that the output voltage of the operational amplifier is incremented by exactly $10 \mathrm{~V}$ when the switch is activated (see Figure 1C). Then, the trimming potentiometers in series with the stage potentiometers are adjusted so that their output voltage is equal to the input voltage divided by 1,000 . When these procedures are performed correctly, the voltage reading on the DVM equals the values that need to be entered on the reference source potentiometers and MSD and sign switches to negate the dc resting voltage of the stage-mounted potentiometers.

Circuit board characteristics and layout can easily degrade the performance of the device. It is advisable to use a printed circuit board with the power supply mounted close to the circuit components (a $1: 1$ scale drawing of the board is available from the authors on request). The inputs of the LH0044C operational amplifier are guarded according to the procedure outlined by the manufacturer (Underwood, 1972). A glass epoxy board should be used to avoid degradation of the input impedance, and sockets for the active components should be avoided since the resistance between pins in the socket can be lower than that of the amplifier. After the components are soldered to ine board, the board should be cleaned with acetone to remove flux, which may exhibit significant conductance, 
resulting in drift. The board should then be coated with an insulating silicone lacquer to insure maintenance of the superior electrical qualities established by these procedures.

\section{Teletype Interface}

Since approximately 500 data points are collected in each brain section, computer processing is a virtual necessity. A simple microprocessor interface encodes the BCD output of the DVM in ASCII on paper tape for further processing on the Ohio State University's Amdahl 470 computer system. (The statistical reduction of autoradiographic data will be described in a later publication.)

The microprocessor interface shown in Figure 3 requires one board microcomputer (RCA Model CDP18S020), an ASR 33 Teletype or its equivalent, a 5-V .25-A power supply, and several integrated circuits that may be wired into an open area of the microcomputer.
The Teletype is easily interfaced to the microcomputer, which comes equipped with a serial interface that accepts a variety of terminal speeds and may be configured for either a 20 -mA current loop or the RS232 interface standard. A batteryoperated circuit for the low-power CMOS memory described in the microcomputer manual allows for retention of programs during power-down periods.

The interface between the Weston DVM and the microprocessor is shown in the top half of Figure 3. This interface was designed so that closure of the switch labeled "print" inputs the most significant four digits of valid data from the DVM into two CDP1852 input ports and raises flag $\bar{E} \bar{F} 2$ on the microprocessor. In operation the switch is debounced by the CD4004B flip-flop. The CD4013 flip-flops synchronize the print signal with the data ready output of the meter and apply a clock pulse to the 1852 input ports. The TTL outputs of the DVM are pulled up with $2.2 \mathrm{~K}$ resistors to make them compatible
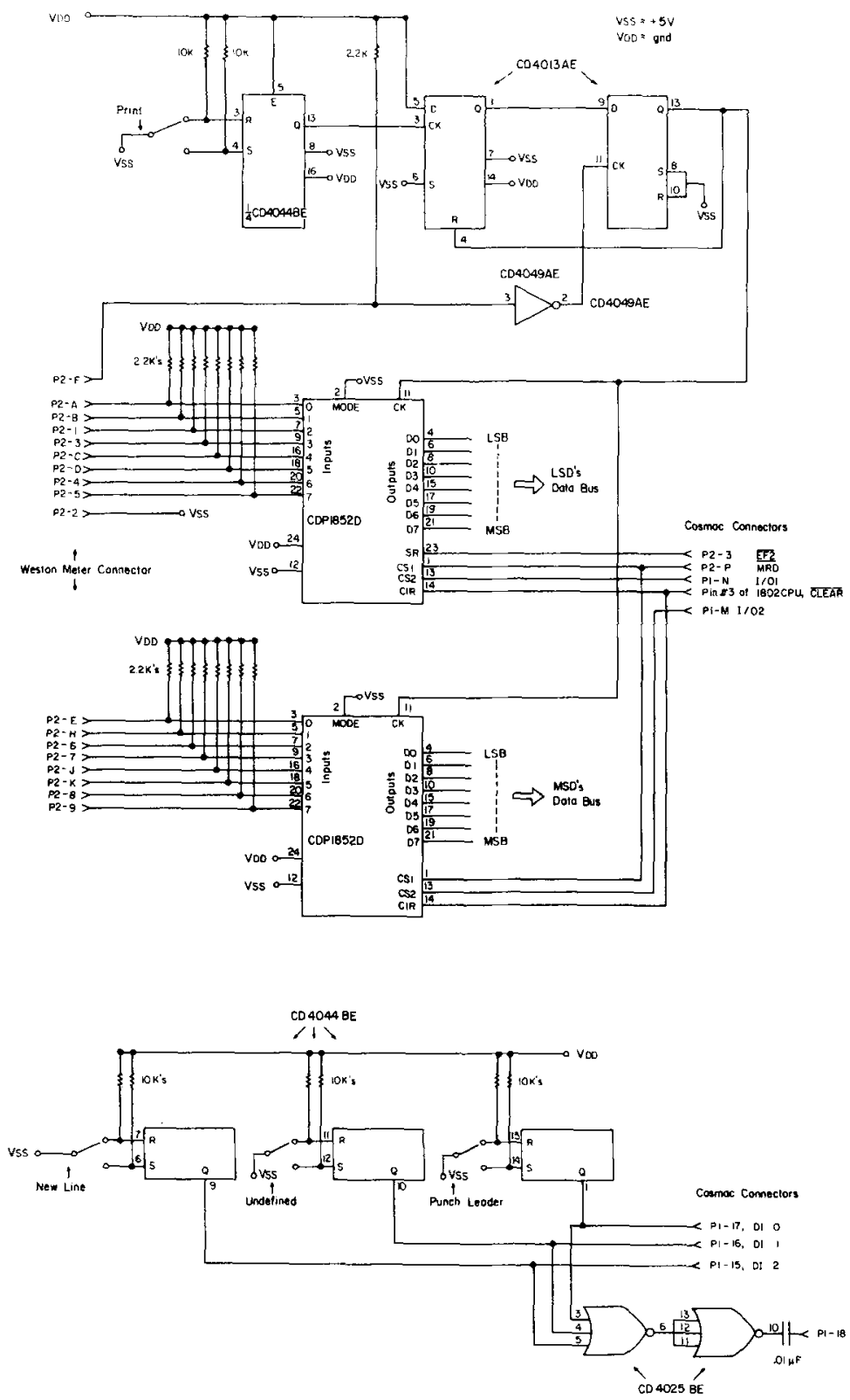

Figure 3. Circuit diagram of interface between Weston DVM and COSMAC 185020 single-board microcomputer. Connector points on left connect to Weston DVM; those on the right connect to COSMAC. 
with CMOS logic requirements. When valid data are clocked into the input ports, the service request line becomes active at flag $\bar{E} \bar{F} 2$ on the microprocessor. The flag line remains high until the contents of the ports are read into the computer, at which time they automatically go low. Read operations are accomplished by simple software $\mathrm{I} / \mathrm{O}$ commands that activate I/O Lines 1 and 2, shown in the schematic. These lines control the three-state outputs of the ports so that data are placed on the data bus at the proper time.

As shown in the bottom half of Figure 3, other switches are wired to the microcomputer. These switches provide formatting of data to correspond with the shape of the nucleus and punching of paper tape at the beginning and end of a run. An additional switch for future expansion is easily added with the already on-board components. For this application, the three remaining flip-flops from the CD4044 debounce three spdt switches. The bounceless outputs are applied to the on-board input port, which has its service request line applied to flag $\overline{\mathrm{E}} \overline{\mathrm{F}} 3$ by inserting Link 4 , as described in the microcomputer manuals. These inputs are applied to a three-input NOR gate. The inverted, differentiated output of the NOR gate is applied to the clock input of the port to signal new data. Upon an $\mathrm{I} / \mathrm{O}$ read command, the data from the port are read into the accumulator, where bit manipulations locate the active input.

\section{Operational Procedures}

Sampling size. With the positioning system described in the preceding section, we analyzed silver grain deposits in a $35 \times 35$ micra area of tissue. ${ }^{1}$ To obtain that specified area, we masked the periphery of a translucent reticule with opaque photographic tape so that light could only pass through a $35 \times 35$ micra area. The reticule then was placed in the ocular of the camera tube, and the photodiode was positioned over the eyepiece and taped to the camera tube with photographic tape. During silver grain measurements, however, the microscope stage is moved in steps of 50 micra. As a consequence, the region sampled by the diode is a $35 \times 35$ micra area centered in the 50 x 50 micra area of movement. Since there is a 15 -micra difference between the 50-micra stage movement and the $35 \times 35$ micra diode sampling area, moving the stage 50 micra instead of 35 micra to another area of tissue is a safeguard against repeated border measurements of adjacent areas. Overlap of adjacent tissue readings can easily occur due to the delicate manual dexterity required to precisely move the microscope stage in 50-micra steps for each measurement. Within the 35 × 35 micra area, however, the millivolt output can be influenced by several manipulations of the micrscope settings, and it is imperative that a rigid protocol be followed in order to avoid possible errors in data collection.

With the exception of the microscope light, the equipment is allowed to warm up for at least $30 \mathrm{~min}$. Since extreme variations in room temperature change the viscosity of the immersion oil as well as the operation of the electrical components, room temperature must be maintained at approximately $72^{\circ} \mathrm{F}$. Below $65^{\circ} \mathrm{F}$, dark-field microscopy is virtually impossible to use in conjunction with this system.

Preliminary settings. As shown in Figure 1E, the binocular eyepiece of the microscope is replaced with an inclined monocular tube equipped with a drawing mirror. Alignment marks have been placed on both the body of the microscope and the base of the monocular tube (Figure 1Ea) so that the tissue image from the tube is aligned with the $X$ and $Y$ movements of the microscope stage. The image of the brain section is projected upon a surface inclined at $13 \mathrm{deg}$ to of fset the parallax caused by the drawing mirror. With a simply constructed slide holder mounted upon the microscope stage, the slide is rotated so that the proper dorsal-ventral orientation can be obtained. Aligning each section in the same orientation facilitates accurate comparison between sections.
The next procedure is to place the plotter paper in the XY plotter such that it fits snugly into the lower left-hand corner. This is important, for later, when the paper is removed for drawing the outline of a specific brain nucleus, it can be replaced exactly in its original position in the plotter. The plotter paper is graphed in 4-mm blocks such that each square represents a 50-micra movement of the microscope stage. Figure 1D illustrates the control knobs of the amplifier modules of the Houston $X Y$ plotter used for coordinating the movements of the microscope stage with the plotter. In our system the inches setting is never changed from "inches" and the calibration knob is never moved from the "cal" position on both axes of the plotter. However, the sensitivity switches for both axes of the plotter initially must be set at zero. Next, with the projected image of the nucleus centered on the inclined projection surface, the plotter pen is moved to any desired position on the plotter paper by turning the zeroing knobs $(\mathrm{Z})$ on the $\mathrm{X}$ and then on the $\mathrm{Y}$ axes.

Coordination of stage with plotter. After these preliminary settings have been made on the plotter, the voltage from the microscope potentiometers is matched with the voltages from the differential amplifiers in the XY plotter to avoid saturation of the plotter amplifiers. Figure 1C illustrates the control apparatus used in our system for carrying out these procedures. The toggle switch at the top of the apparatus is pushed to X. The subsequent voltage output displayed on the DVM is the value entered into the $\mathrm{X}$ adjustable voltage source by moving the $X$ toggle switches and the $X$ digital potentiometers to the appropriate voltage value. This voltage represents the resting potential on the $X$ axis of the stage, and when entered into the $X$ adjustable voltage source, it acts as a bucking voltage on the plotter differential amplifier (see Figure 2). For example, if the voltage on the meter is +103.94 , the first toggle switch is moved to the "plus," the second is moved to " 1 ," and " 03.9 " is punched into the $\mathrm{X}$ digital potentiometer, ignoring the last digit. All of the procedures are repeated for the $\mathrm{Y}$ axis settings, taking only a few moments for the entire calibration. The final step in coordinating the microscope stage and plotter pen movements involves turning the $\mathrm{X}$ and $\mathrm{Y}$ sensitivity knobs from 0 to the $100-\mathrm{mV} / \mathrm{in}$. range on the plotter (Figure 1D). The $X$ and $Y$ zero knobs $(Z)$ are then repositioned if desired. The potentiometers on the microscope stage and the differential amplifiers in the $X Y$ plotter are coordinated. When millivolt readings are eventually taken, the graphed plotter paper serves to guide the experimenter's movements of the microscope stage.

Drawing the nucleus. After the stage and the $X Y$ plotter are coordinated, the nucleus is drawn on the plotter paper so that its boundaries are defined to the experimenter as he moves the microscope stage and, consequently, the pen on the plotter. Thus, by merely watching the excursion of the plotter pen travel throughout the drawn outline of the nucleus, the experimenter is aware of the exact location in the nucleus that the photodiode is sampling.

Several steps are involved in drawing the nucleus accurately. First, oil is placed on the slide to control for its refractive index through all ranges of magnification. Oil is used in contact only with the $100 \mathrm{X}$ oil objective; it should never touch the lenses of the lower power objectives. Next, under low-power light field, an outstanding landmark is located in the vicinity of the area to be read by centering the landmark over a fixed dot on the white projection surface. The landmark's position is marked by the XY plotter pen on the paper. This landmark is then relocated over the fixed dot under an intermediate power objective to facilitate finding it once more under $100 \mathrm{X}$ oil immersion. After the landmark has been relocated with the oil objective, its location is marked again on the paper with the plotter pen, and the "high-power mark" is differentiated from the "low-power mark" by an arrow. Two more landmarks are identified using the same procedures. As a guard against possible 
error during the landmark procedures, the distance and direction between the respective high-power and low-power marks for all landmarks are checked to make sure they are identical. After all landmarks have been properly identified, the paper is removed from the plotter in preparation for drawing the outline of the nucleus. At this point, due to differences in scaling, the projection surface must be raised $25 \mathrm{~mm}$. The tissue is then refocused on the white angled projection surface with the lowpower objective. This modification of projection surface height insures that the three high-power marks found under oil will align with the same three landmarks of the low-power reflected image. Since the image of the nucleus reflected from the mirror onto the inclined surface on the table is reversed from the image through the binocular eyepiece and thus to the photodiode, it is necessary to reverse the plotter paper before drawing the outline such that the top of the page is now the bottom. The high-power marks on the plotter paper are then aligned with the original landmarks on the reflected image, and the outline of the nucleus is drawn. When completed, the paper is replaced in the XY plotter right side up so that it again fits flush in the lower left-hand corner.

Dark-field condenser calibration. At this stage in the procedure, the monocular tube with mirror is replaced with the binocular eyepiece and the attached photodiode. Like the monocular tube, the binocular eyepiece assembly is matched with the body of the microscope with the aid of alignment marks. Under light-field oil immersion, an area of relatively high silver grain density is focused upon, and the light-field condenser is replaced with the dark-field oil condenser.

Several precautions should be taken with the dark-field condenser settings to assure that millivolt readings are comparable from one section to another. The potential sources of variability are the angle of light through the dark-field condenser, the focus of the tissue through the microscope objectives, and the height of the condenser. First, the dark-field condenser knobs, which adjust the light angle, are set permanently such that a uniformly dark field is obtained. It is important that the background illumination under dark field be consistent from reading to reading and from slide to slide. The net consequence of varying the contrast of figure (grains) to ground (unlabeled tissue) could be that the millivolt-to-grain slope of +.01 reported by Hughes et al. (1977) would change. Permanent fixture of the dark-field centering screws in a position that achieves a uniformly dark field negates this figure-ground contrast problem.

As an interim experiment, we investigated the consequences of varying the focus of the tissue under high-power oil dark field upon the millivolt readings. Since brain sections are not precisely flat, and since there are slight variations in section thickness, or imperfect coating of slides with emulsion, slight variations in focal planes within a section could occur. Refocusing the section is not necessary, but corrections of gross aberrations should be made. As shown in Figure 4A, slight focal changes do not induce significant variability in millivolt readings. Multiple readings of 25 data points, each representing a $35 \times 35$ micra area, were collected through a lateral geniculate nucleus. These 25 readings were repeated under the different focusing conditions. Thus, data were collected when the section was optimally focused ( $0 \mathrm{deg}$ on the fine-focus knob) and when it was $1 \mathrm{deg}$, $2 \mathrm{deg}$, and $4 \mathrm{deg}$ out of focus. As seen in Figure $4 \mathrm{~A}$, variations in focus do not influence repeated millivolt readings.

The last lighting problem is related to the height of the darkfield condenser. Hughes et al. (1977; see Footnote 1) found that
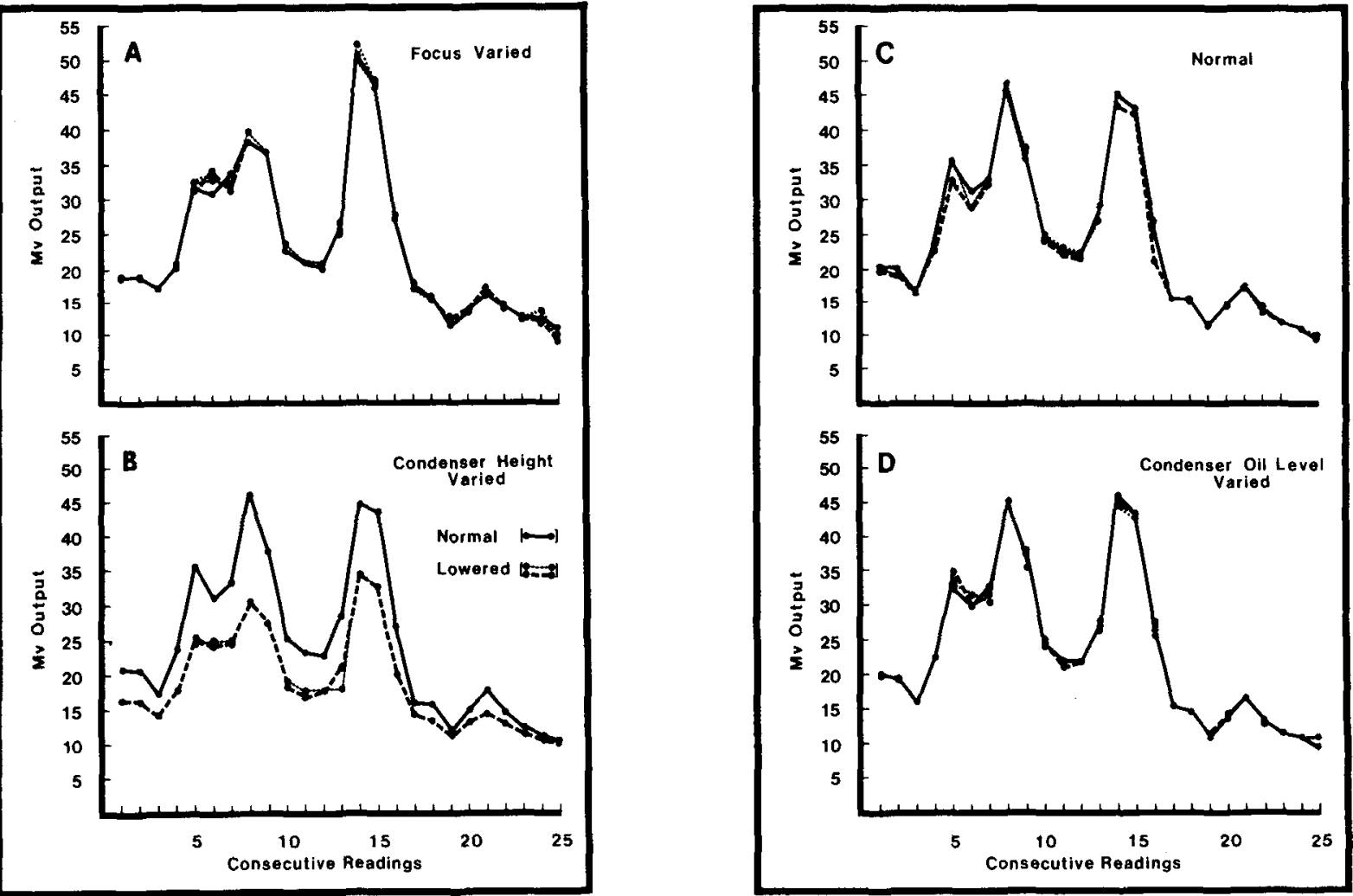

Figure 4. (1) Multiple readings with focus at optimal level, and $1 \mathrm{deg}, 2 \mathrm{deg}$, and $4 \mathrm{deg}$ out of focus as determined by the finefocus dial on the microscope. (B) Multiple readings with condenser at normal and grossly abnormal (lowered) level. Note the nonalgebraic relationship between the two conditions; that is, the larger the millivolt reading, the greater the difference between the two settings. (C) Four separate readings were taken following normal operating procedures. Between each series of readings the darkfield condenser was lowered and cleaned, fresh immersion oil was applied, and then the condenser was raised to operating height following the procedures previously described. (D) Multiple readings (four) taken with immersion oil at significantly increased and decreased levels. 
varying the height of the dark-field condenser by the small degrees typical of normal operating procedures changes the $y$ intercept of the millivolt-to-grain correlation, but the slope of the line remains parallel to the original line. To eliminate this variation of the $y$ intercept, we adopted the following procedures. Typically, there are two condenser positions that achieve a good dark field. The first occurs immediately after the oil contacts the underside of the slide. This height is used because the second dark-field position, which occurs much closer to the slide, produces a much brighter overall background and inflates the millivolt readings. The first dark-field condenser height is estimated by a mark on the microscope. The mark serves as a guide to obtain the same condenser height for other sections. As seen in Figure 4B, however, gross variations in condenser height result in changes in both slope and $y$ intercept of the millivolt-to-grain correlation and must be avoided. Yet, as Figure $4 \mathrm{C}$ shows, repeated readings using the first dark-field condenser height yield very consistent and comparable data. Because the amount of immersion oil can also affect the condenser height when the condenser reaches the first dark field, the amount of oil placed on the condenser was varied. In one series through 25 readings, very little oil was applied. In a second series, the normal amount (in Figure 4C) was applied; in a final series, significantly more oil than normal was placed upon the condenser. Varying the quantity of oil on the condenser had virtually no effect on the millivolt readings (Figure $4 \mathrm{Cd}$ ). With the three condenser variables at known settings, a consistently good dark field can be obtained from section to section and from slide to slide, and with very little adjustment.

Accounting for background noise. To preserve the correlation between millivolt readings and number of grains reported in the Hughes et al. (1977) study, one final manipulation was performed. The lowest background grain area on the tissue is located by passing the light image through the binocular eyepiece on the microscope to the photodiode. The millivolt output read from the DVM reflects the intercept value for the section in question, and by either adding or subtracting a constant, the millivolt reading can be set with the zero screw on the control box to the same $+2.7-\mathrm{mV}$ intercept originally reported by Hughes et al. (1977; see Footnote 1). However, the amount of label injected, the survival time of the animal after injection, and the exposure time of the autoradiographs should be constant. With the present equipment, background noise in general can be accounted for, and all readings from section to section can be made comparable. However, this procedure cannot be used to compensate for poor histology in which high background labeling is a problem.

Reading the nucleus. After the plotter has been coordinated with the movements of the microscope stage and the photodiode has been calibrated, the nucleus under study is ready for scanning. A short assembly language routine is used to format data on the Teletype and punch paper tape for later processing. At the beginning and end of a session, the punch leader switch is closed, resulting in a string of 180 nulls. Data are entered sequentially from left to right by moving the stage while observing the pen's position on the plotter and pressing the print switch for each new reading. After 10 data points are entered, the microprocessor automatically initiates a carriage return and line feed on the Teletype.

A new row in the nucleus is signified by closure of the new line (Figure 3) switch, which causes the microprocessor to output a carriage return and two line feeds. The extra line feed provides a simple means for later determination of the origin of each data point and places a character on the paper tape for computer recognition of the new row. The cartographic programs used to reduce data require that data be entered in the form of a matrix. To accommodate this requirement, an imaginary origin for the $\mathrm{X}$ axis is assumed at the beginning of each scanning session. At the start of each new row, the microcomputer waits for the entry of a two-digit decimal number signifying the number of $\mathrm{X}$ intervals from the origin to the beginning of the nucleus. It then prints that number of zeroes to provide the correct starting $\mathrm{X}$ coordinate. This easy compensation for the irregular shape of brain nuclei also reduces software overhead in later processing. (An assembly listing of this program that requires .25K RAM is available from the authors on request.)

\section{RESULTS AND DISCUSSION}

Hughes et al. (1977) report that varying the dark-field condenser height only raises (or lowers) the y intercept of the straight-line relationship between millivolt readings and the number of grains counted by eye. These results suggest that the same slope describes the relationship between millivolt grains from section to section, but in that study, the effect of constant condenser settings was not empirically tested from section to section, slide to slide, and animal to animal. In the Hughes et al. study, only one row through one section of a nucleus was analyzed. After we standardized the procedures for achieving constant dark-field condenser settings and for maintaining the y-intercept value of $+2.7 \mathrm{mV}$ as reported by Hughes et al., we collected various millivolt readings over several sections in different animals to determine whether the slope of the relationship of millivolts to grains remained the same as that reported by Hughes et al. To accomplish this recalibration, grain counts by two independent observers were averaged, and millivolt readings from the photodiode were collected over the same area. Samples from high-, medium-, and low-grain regions were collected, without correcting the height of the condenser, from a number of different sections and animals. The slope of the millivolt-grain relationship remained the same as that reported by Hughes et al. (slope $=.011, \mathrm{SEM}=.001$ ), within the same error for interobserver agreement. Therefore, if two sections of readings have the same slope and the intercept of the line is an arbitrary function of the condenser settings, all sections can be standardized as stated in the Method section of the present paper. With the equipment described here and with all condenser settings held constant, all readings can be made comparable by finding the lowest background area and then adjusting the DVM to $+2.7 \mathrm{mV}$. In this way, differences in magnitude of labeling as reflected by millivolt output can be preserved as they vary from section to section. Raw scores from one animal are directly comparable to another, provided factors such as the amount of injected label, the survival time after injection, and the exposure times of the material are constant.

Quantitative treatment of anatomical data is a new but important step for determining the effects of 
particular treatments on connectivity within the brain. Rapid advances in solid state technology allowed for the design of a relatively inexpensive system for rapidly collecting large amounts of quantitative anatomical data.

\section{REFERENCES}

Hughes, H. C., Meyer, P. M., Meyer, J. W., Meyer, D. R., \& Bresnahan, J. C. An inexpensive microphotometer system for measuring silver grain densities in autoradiographs. Stain Technology, 1977, 52, 79-84.

UNDERWOOD, R. K. New design techniques for FET operational amplifiers (AN-63). In M. K. Vander Kooi (Ed.), Linear applications (Vol. 1). Santa Clara, Calif: National Semiconductor, 1972.

\section{NOTE}

1. Hughes et al. (1977) reported that measurements were made over regions of tissue 256 square micra ( $16 \times 16$ micra). The size of that area was reported incorrectly. Actually, the millivolt readings and grain counts were sampled over regions 1,225 square micra ( $35 \times 35$ micra), the same as in the present study.

(Received for publication November 15, 1978; revision accepted April 12, 1979.) 